山्山FFANÇAISE

$>\mathrm{DE}$

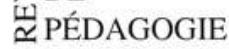

Revue française de pédagogie

Recherches en éducation

171 | avril-juin 2010

La mixité scolaire : une thématique (encore)

d'actualité?

\title{
La mixité, une évidence trompeuse ? Entretien avec Martine Chaponnière, Université de Genève propos recueillis par Françoise Vouillot
}

\section{(2) OpenEdition}

\section{Journals}

\section{Édition électronique}

URL : http://journals.openedition.org/rfp/1905

DOI : 10.4000/rfp. 1905

ISSN : 2105-2913

Éditeur

ENS Éditions

Édition imprimée

Date de publication : 1 juillet 2010

Pagination : 69-75

ISBN : 978-2-7342-1187-7

ISSN : 0556-7807

\section{Référence électronique}

"La mixité, une évidence trompeuse ? Entretien avec Martine Chaponnière, Université de Genève ", Revue française de pédagogie [En ligne], 171 | avril-juin 2010, mis en ligne le 01 juin 2014, consulté le 19 avril 2019. URL : http://journals.openedition.org/rfp/1905; DOI : 10.4000/rfp.1905 


\section{La mixité, une évidence trompeuse? Entretien avec Martine Chaponnière, Université de Genève}

\section{Pouvez-vous nous résumer les débats qui ont pris place en Suisse lors de l'introduction de la mixité ?}

Lorsqu'elle fut introduite de manière plus ou moins généralisée dans toutes les écoles secondaires européennes dans le courant des années soixante et soixante-dix, la mixité était censée réaliser l'égalité des chances entre filles et garçons et il ne semblait faire aucun doute qu'elle ne produirait que des effets bénéfiques pour tous. Or tous les constats montrent qu'elle s'est aussi accompagnée d'effets pervers.

En Suisse comme en France, il n'y a pas eu de débat sur l'introduction de la mixité au niveau secondaire. Au niveau primaire, en revanche, un problème a surgi dans le canton de Vaud. En effet, étant donné les programmes différents suivis par les garçons et les filles, des barèmes différents étaient aussi appliqués pour l'examen d'entrée au collège (qui donne accès à l'université). En gros, pendant que les garçons faisaient des mathématiques et du français, les filles cousaient. Les barèmes des examens de français et de mathématiques étaient donc moins sévères pour les filles. Lorsque les programmes devinrent les mêmes pour les deux sexes, plus de filles que de garçons réussissaient les examens d'entrée au collège, ce qui ne manqua pas d'inquiéter les autorités scolaires. Celles-ci gardèrent l'idée du barème différencié, mais l'adaptèrent à la nouvelle situation, en relevant les exigences vis-à-vis des filles par rapport à celles imposées aux garçons.

En 1981, le peuple suisse votait un article de la Constitution fédérale garantissant l'égalité des droits entre femmes et hommes, notamment dans l'éducation. C'est ce qui permit à un certain nombre de parents dont les fillettes étaient mises en échec par l'inégalité du barème de demander un recours contre ce système discriminatoire. Cette année-là, 1211 filles et 1181 garçons ont été admis au niveau secondaire. Si les barèmes avaient été les mêmes pour les deux sexes, 42 filles ayant raté auraient réussi et 84 garçons ayant réussi auraient échoué aux examens. II y aurait alors eu $55 \%$ de filles et $45 \%$ de garçons dans la filière "noble ", proportion apparemment inadmissible pour les autorités scolaires. Pour ces dernières en effet, sans barèmes différenciés, les filles « entreraient en surnombre à l'école secondaire, privant par là même un certain nombre de garçons - soit tout un groupe de la population - de la possibilité d'acquérir une formation à laquelle ils doivent, dans la structure actuelle de notre société, pouvoir accéder, si l'on ne veut pas créer un déséquilibre préjudiciable à tout le monde " (Tribunal fédéral, 1982). Pour les parents des fillettes mises en échec, ce fut une longue bataille, mais finalement les quelques parents qui ne s'étaient pas découragés en cours de route eurent gain de 
cause et les barèmes sont aujourd'hui les mêmes pour les deux sexes. Cet exemple pose d'ailleurs un problème délicat en matière de discrimination positive. Argumentant contre le recours des parents, les autorités vaudoises affirmèrent que « le principe fondamental de l'égalité de traitement entre hommes et femmes [...] n'empêche pas le constat de différences entre les sexes, notamment entre les élèves filles et les élèves garçons de onze ans environ, les filles bénéficiant à cet âge d'une certaine avance sur leurs camarades masculins ». Mais au final, le tribunal fédéral rendit en 1982 un jugement en faveur des parents des fillettes : «Chaque élève doit pouvoir se prévaloir individuellement de l'égalité de traitement juridique, indépendamment de son appartenance à l'un ou l'autre sexe. » La problématique posée ici est celle de l'égalité des chances vs l'égalité des droits. $\mathrm{Si}$ effectivement filles et garçons ne suivent pas le même développement psychologique et intellectuel au moment de l'adolescence, la question doit être posée soit de faire des classes séparées, soit de faire des pédagogies différenciées.

\section{Vous avez évoqué des " effets pervers" de la mixité. Pourriez-vous nous dire lesquels en particulier?}

L'introduction de la mixité s'est accompagnée de la mise en lumière, dans la recherche scientifique, des comportements différenciés du corps enseignant vis-à-vis des filles et des garçons. Depuis la note de synthèse très complète à ce propos publiée par Marie Duru-Bellat (1995a), rien de vraiment probant n'est venu infirmer les résultats de recherche présentés. En résumé, les enseignants accordent plus d'attention aux garçons (en moyenne, deux fois plus), ce qui se manifeste de plusieurs manières. Maîtres et maîtresses interrogent plus souvent les garçons, les encouragent plus mais aussi les critiquent plus. Ils et elles ont aussi des attentes plus élevées vis-à-vis des garçons que des filles, ce qui apparaît paradoxal vu la meilleure réussite scolaire des filles et qui pourrait s'expliquer par le fait que les enseignants considèrent les garçons comme des "sous-réalisateurs" qui pourraient faire beaucoup mieux s'ils s'en donnaient la peine (Duru-Bellat, 1995b). Les enseignants interrogent les filles pour rappeler la leçon précédente et les garçons pour la production de savoir (Mosconi, 2003). Les interactions varient aussi en fonction de la discipline, selon qu'elle est connotée féminine ou masculine. Enfin les enseignants attendent des filles qu'elles coopèrent au bon fonctionnement et à la bonne ambiance de la classe et les utili- sent comme "auxiliaires pédagogiques » (Zaidman, 1996). Ces différents résultats méritent bien sûr d'être nuancés selon le degré étudié mais, dans l'ensemble, le traitement des enseignants tend à valoriser les garçons et, dans une moindre mesure, à dévaloriser les filles ou à mobiliser envers elles les stéréotypes du féminin : «Le contexte de la mixité, en rendant le sexe saillant, en développant chez les élèves des processus de catégorisation sexuée et de stéréotypage, tend à accentuer le caractère asymétrique des catégorisations de sexe, valorisant ce qui est masculin et dévalorisant tout ce qui est féminin. " (Mosconi, 1999) Ainsi les filles seraient plus ou moins bien «absorbées» dans un système masculin. Comme a pu l'écrire une journaliste du Times en 1973, peu après la généralisation de la mixité : «Trop d'écoles sont des écoles de garçons avec des filles dedans. »

\section{Ainsi, selon vous, serait donc à l'œuvre dans les classes cette "valence différentielle des sexes " (Héritier, 1996). Quelles en sont les conséquences en ce qui concerne l'estime de soi des filles?}

L'estime de soi dépend de plusieurs facteurs, parmi lesquels la prégnance des stéréotypes de sexe peut jouer un rôle. Par exemple, dans un groupe d'étudiants en mathématiques choisis parmi les meilleurs et disant tous aimer cette discipline, les résultats aux tests d'admission à l'université varient selon le message préalablement transmis. Dans l'expérience en question, les expérimentateurs avaient dit à une moitié du groupe que les filles réussissaient moins bien dans ces examens et à l'autre moitié qu'il n'y avait pas de différences perceptibles dans les résultats entre garçons et filles. Comme on pouvait s'y attendre, les filles du deuxième groupe ont réussi aussi bien que les garçons, alors que les performances des filles du premier groupe sont tombées à moins de la moitié de celles des filles du deuxième groupe. Les auteurs de l'expérience interprètent cette chute des performances par le fait que la remarque sur l'interdépendance entre le sexe et le résultat renverrait les filles au stéréotype négatif selon lequel les femmes seraient moins douées pour les mathématiques. C'est ce qu'ils appellent le « stereotype threat », la menace du stéréotype, ainsi définie : "La menace du stéréotype est activée lorsqu'un individu court le risque de confirmer comme une caractéristique personnelle un stéréotype peu flatteur ou négatif généralement appliqué au groupe auquel il ou elle appartient. » (Spencer, Steele \& Quinn, 1999) 
De nombreuses recherches, américaines surtout, confirment l'existence de la menace du stéréotype sur les performances de groupes stigmatisés. Reste évidemment à savoir quel rôle exactement elle joue parmi d'autres variables ayant une incidence sur les performances. II n'en reste pas moins que les divers résultats de recherche dont nous avons fait état ici montrent que, si la mixité va aujourd'hui de soi, elle mérite tout de même d'être questionnée dans les effets imprévus qu'elle a provoqués. Dans un contexte européen laïque et républicain, il est difficile d'imaginer un retour, même partiel, à la non-mixité.

\section{Y a-t-il, dans les pays que vous connaissez le mieux, des débats quant à l'opportunité d'un retour à la non-mixité ?}

Récemment, des voix se sont élevées, en Suisse alémanique surtout, pour dénoncer une mixité qui serait plus défavorable aux garçons qu'aux filles. II s'agit en Suisse de quelque chose de nouveau, mais cette approche existe depuis longtemps au Québec, lancée par les mouvements dits "masculinistes". En Suisse, un quotidien grand public titrait en octobre 2008: "L'école discrimine les garçons". Le cri d'alarme était lancé par deux hommes assez connus : le pédiatre et ancien professeur à l'hôpital des enfants de Zurich Remo Largo, considéré dans les pays germanophones comme "la " référence en matière de développement de l'enfant, et le psychologue Allan Guggenbühl, auteur d'un ouvrage intitulé Kleine Machos in der Krise [Petits machos en crise]. Ces auteurs s'inquiètent du fait que les garçons forment la majorité des classes spéciales avec enfants à problèmes (dyslexie, troubles du comportement, troubles psychomoteurs, etc.) et du fait que, plus la filière est faible, plus on y trouve de garçons, dont les mauvais résultats scolaires doivent nous préoccuper. Si ce constat est dans l'ensemble juste, il faut néanmoins le relativiser. L'enquête PISA a montré qu'en Suisse, les filles obtiennent de moins bons résultats que les garçons dans les branches scientifiques. Des pays limitrophes et de ceux ayant de meilleurs résultats, la Suisse est le seul à présenter une différence statistique significative des résultats entre les genres pour les sciences.

Pourquoi sont-ce les garçons qui font problème à l'école? Dans le sillage des masculinistes québécois, ces auteurs partent de l'idée que l'école est devenue une sorte de biotope féminin duquel aurait disparu tout esprit de compétition et de confrontation (suppression des notes, travail de groupe, recherche d'harmonie, etc.). Or la situation de défi, intellectuel ou physique, constitue un rouage crucial d'apprentissage chez les garçons. Ce ne seraient donc pas les garçons qui posent problème, mais leur environnement qui ne respecterait pas les caractéristiques de la masculinité. Relativisons la position de ces auteurs. Le décrochage scolaire y apparaît comme un phénomène nouveau alors que la prédominance féminine, en particulier en primaire, existe depuis longtemps. II apparaît donc difficile de corréler les deux phénomènes de manière aussi simpliste. En outre, ce que ces auteurs ne disent pas, c'est que deux problèmes parallèles coexistent: d'une part le comportement problématique des garçons pour lesquels la réussite scolaire - et pas seulement l'école - relève du féminin, d'autre part la moindre estime de soi des filles (Duru-Bellat, 1995b), malgré leurs meilleures performances. Les stéréotypes de sexe (filles « appliquées » et garçons "turbulents") restent dans une certaine mesure valables et il vaut la peine d'en tenir compte plutôt que de les nier.

À l'école, en tout cas en Suisse romande, plus influencée par le contexte français pour lequel tout retour à la non-mixité serait un pas en arrière, il n'existe pas à notre connaissance d'organisation d'activités scolaires non mixtes. En revanche, le Bureau de l'égalité entre les femmes et les hommes du canton de Vaud a, avec le soutien du département de l'instruction publique, publié, sous l'intitulé L'école de l'égalité, quatre manuels en fonction des différents degrés du primaire, pour aider les professeurs à dispenser un enseignement qui permette aux filles et aux garçons de se réaliser dans l'égalité, en respectant leurs caractéristiques propres " afin que les filles, comme les garçons, puissent se développer au mieux de leurs capacités, en fonction de leurs désirs propres et indépendamment des préjugés de sexe" (Bureau de l'égalité entre les femmes et les hommes du canton de Vaud, 2006). Le problème est que le recours à ces fascicules est laissé au bon vouloir du corps enseignant et que nous ne disposons pas de données sur leur utilisation.

\section{Pouvez-vous nous donner quelques expériences pilotes de non-mixité ?}

En Suisse alémanique, l'école primaire publique Steinacker à Pfäffikon (canton de Zurich) a tenté en 2008 de séparer filles et garçons pendant sept semaines dans les cours de langues, mathématiques et travaux manuels. L'expérience s'est révélée très positive pour tout le monde (corps enseignant et 
élèves), au point que l'école songe à instituer des enseignements séparés pour certains domaines. Mais ce type d'expériences reste rare. II existe en Suisse une quinzaine d'écoles non mixtes, la plupart privées et majoritairement pour filles. Des tentatives plus informelles existent aussi en Suisse alémanique, comme le Réseau de travail scolaire pour garçons. Ce réseau est né dans les années 2000 à la suite de graves problèmes de viols collectifs et de violences commises par des garçons assez jeunes. Ce sont des hommes qui interviennent dans les classes pour proposer aux garçons une image positive du masculin et de la virilité. Mais le travail de ce réseau ne va pas dans le même sens que celui proposé par Guggenbühl, ce professeur de psychologie évoqué plus haut, auteur d'un ouvrage sur les « petits machos en crise". Celui-ci soutient que les garçons doivent pouvoir vivre leur goût du défi et de la bagarre, doivent pouvoir développer leur vraie personnalité de garçons pour pouvoir affronter l'école dans des conditions qui leur conviennent. L'approche du Réseau de travail scolaire pour garçons est différente : lors des activités qu'il organise, les garçons sont amenés à remettre en question les idéaux masculins violents et s'entraînent à la résolution non violente des conflits. La ville de Munich a institué un poste de délégué aux jeunes gens (au vu du décrochage scolaire des garçons), qui semble travailler avec la même approche.

En Suisse alémanique encore, fortement influencée par ce qui se fait en Allemagne, l'accent est mis sur le «Mädchenarbeit », le travail avec les jeunes filles. Le constat de départ est que les filles sont confrontées à une image type de la jeune fille moderne, l'image d'une adolescente dotée d'une bonne estime de soi, maligne, intelligente, éduquée, sexy, cool, autonome, genre Madonna. À cela s'ajoute l'idée qu'elles sont les égales des hommes. Or, pour bien des adolescentes, cet idéal-type ne correspond pas à ce qu'elles vivent dans la réalité. Le Mädchenarbeit consiste donc à permettre à ces jeunes filles de renforcer leur confiance en elles, d'augmenter leur estime de soi, de s'orienter dans les questions de pauvreté, de migration, d'orientation et de formation professionnelle, de conciliation de vie familiale et professionnelle, de santé (notamment les problèmes nutritionnels) et de participation à la vie locale associative et civique.

En Suisse romande, dans les centres de loisirs, les travailleurs sociaux ont de grosses inquiétudes car les filles ne viennent plus. Cet absentéisme est dû aux violences sexistes (verbales ou physiques) qu'elles subissent et au fait que les garçons monopolisent l'espace et les installations (jeux, parc informatique) et qu'elles ont beaucoup de peine à y avoir accès. Finalement les adolescentes s'excluent d'elles-mêmes de ces ambiances machistes, basées sur des rapports de force et sur un langage vulgaire et irrespectueux. Les choix des maisons de quartier sont divers pour faire revenir les filles : certaines organisent des activités non mixtes (pour filles seulement), d'autres tentent d'inculquer aux garçons le respect de l'autre et des filles en particulier (GRAAV, 2008). Les différents exemples évoqués montrent que la mixité n'est au fond remise en question que lorsqu'elle pose véritablement problème, comme dans les centres de loisirs où s'établit de facto une non-mixité. Les activités non mixtes sont organisées dans le parascolaire (réseaux pour garçons et réseaux pour filles) mais, dans le champ scolaire proprement dit, il n'est, à quelques rares exceptions près, pas question de tenter des expériences de non-mixité. Ce vers quoi on semble se diriger, ce serait plutôt des pratiques pédagogiques non sexistes.

\section{Derrière ces expériences, y a-t-il volonté de promouvoir des didactiques différenciées et si oui, quels problèmes cela pose-t-il à vos yeux?}

Appliquer une pédagogie non sexiste se fonde sur la notion de pédagogie de la diversité, une pédagogie non plus centrée sur un individu neutre, mais sur des individus membres de groupes sociaux, ethniques, religieux et de sexe. En ce qui concerne ce dernier point, la question qui se pose est alors celle-ci : dans une pédagogie de la diversité, faut-il imposer aux enseignants une formation sur les questions du genre? Les réticences sont évidemment nombreuses dans la profession, dont une large part considère l'égalité des sexes comme un problème réglé. En outre, la majorité des enseignants se refusent à tenir compte, dans leur pratique, des différences de genre, par crainte d'apparaître sexistes. Et avec quelque raison: le danger existe en effet de retomber dans une logique stéréotypée en voulant identifier d'abord, et prendre en compte ensuite les différences (modes d'apprentissage, comportements) entre les deux sexes.

Une brochure publiée par deux chercheuses bâloises (Baur \& Marti, 2000) s'est donnée pour tâche d'élaborer les bases d'un enseignement non sexiste, identifiant au préalable les modes d'être des femmes et des hommes adultes en situation de formation continue. Par exemple, les femmes discutent de manière plus 
émotionnelle que les hommes ou elles préparent plus leurs interventions. Les hommes quant à eux sont plus sûrs d'eux, sont de meilleurs orateurs et tiennent souvent des monologues. Les auteurs précisent bien qu'il ne s'agit pas là d'attitudes liées au sexe biologique mais qu'elles remontent à la division traditionnelle du travail entre les genres établie au $\mathrm{XIX}^{\ominus}$ siècle, et que ces attitudes participent du doing gender. Rappelons que la théorie du doing gender (West \& Zimmerman, 2009) établit la catégorie du genre comme mise en acte: «Plutôt que comme une propriété des personnes, nous concevons le genre comme un trait émergent des situations sociales: [...] le genre d'un individu n'est pas seulement une dimension de ce qu'il est ; il est, plus fondamentalement, quelque chose que l'on fait, et que l'on fait de manière répétée, en interagissant avec autrui. "Malgré les précautions des auteurs de la brochure sur un enseignement non sexiste pour éviter de tomber dans le "tout inné ", on peut quand même craindre que, sans une claire conscience des théories du genre, ce type de manuel risque de conforter les stéréotypes de sexe plutôt que de les affaiblir. Nous sommes ici dans le domaine de l'éducation des adultes, mais la question fondamentale reste la même pour le champ scolaire : comment " tenir compte " des différences de genre sans les renforcer? Comme le souligne la pédagogue allemande Doris Lemmermöhle, lorsqu'il s'agit de démanteler les stéréotypes de sexe et les inégalités, les enseignants se trouvent face à des exigences contradictoires : ils doivent en même temps thématiser les rapports de sexe et ne pas les exacerber; ils doivent tenir compte de l'appartenance de sexe des élèves sans les y réduire ; ils doivent mettre au jour les règles et les mécanismes de production des différences et des hiérarchies de sexe, au risque de les produire eux-mêmes ; ils doivent contribuer à la compréhension des rapports de sexe, tout en étant euxmêmes imbriqués dans l'ordre symbolique des sexes !

II existe néanmoins quelques pistes à suivre pour des pratiques d'enseignement respectueuses du genre. Par exemple, dans l'enseignement de la physique, discipline connotée masculine, il n'est pas superflu de tenir compte des intérêts des filles, de leur confiance en elles par rapport à cette matière et de la compréhension préalable qu'elles ont des éléments qui la constituent. Une recherche expérimentale dans les écoles bernoises (Herzog et al., 1998) montre qu'à quinze ans, presque tous les garçons ont démonté au moins une fois un appareil électrique et parcouru ou lu une revue technique, ce qui n'est pas le cas des filles. Après avoir questionné les filles et les garçons sur leurs motivations et leurs champs d'intérêt en physique, l'équipe de recherche a construit un enseignement qui tienne compte des intérêts des filles. Au final, les résultats des filles ont été moins performants dans les groupes de contrôle et ceux des garçons n'ont pas été moins bons dans le groupe expérimental. À notre connaissance, cette expérience n'a pas été reprise ni même utilisée par la suite.

\section{Conviendrait-il de former le corps enseignant à ces questions ?}

Comme nous l'avons évoqué plus haut, la formation du corps enseignant en matière de genre est plus que lacunaire. II n'y a pas, en Suisse, de véritable politique de l'égalité dans le champ scolaire. Les germanophones, de leur côté, bénéficient de plusieurs guides pour s'autoformer dans ce domaine. Mais en territoire francophone, c'est la disette. En France, la Convention interministérielle pour l'égalité des chances entre les filles et les garçons, reconduite en 2006 et valable jusqu'en 2011 (Ministère de l'Emploi, de la Cohésion sociale et du Logement et al., 2006), prévoit trois axes :

- améliorer l'orientation scolaire et professionnelle des filles et des garçons pour une meilleure insertion dans l'emploi ;

- assurer auprès des jeunes une éducation à l'égalité entre les sexes ;

- intégrer l'égalité entre les sexes dans les pratiques professionnelles et pédagogiques des acteurs et actrices du système éducatif.

C'est évidemment ce troisième point qui nous intéresse ici et il n'est pas surprenant d'apprendre que c'est celui qui a été le moins mis en œuvre jusqu'à présent. II existe bien quelques modules dits de "sensibilisation" mais ils sont facultatifs, de courte durée (trois à six heures), le plus souvent offerts dans le cadre de la formation continue. Leur mise en œuvre repose en général sur la volonté d'acteurs très motivés. Sans doute la résistance du corps enseignant y est-elle pour beaucoup. C'est pourtant à notre sens en reconnaissant les différences qu'on peut produire de l'égalité, non en les niant. Le danger est évidemment, comme nous l'évoquions plus haut, que cette reconnaissance de la différence renforce les stéréotypes plutôt qu'elle ne les affaiblisse, que cette prise en compte du genre se traduise par une pratique du doing gender telle que décrite par West et Zimmermann (2009).

Pour éviter ce piège, une véritable formation des enseignants doit être mise sur pied, qui leur donnerait une "compétence de genre » entendue comme suit : 
« La compétence de genre englobe le savoir relatif aux rapports sociaux de sexe et à leurs causes ainsi que la capacité d'appliquer ce savoir dans la vie quotidienne [...]. Utiliser la compétence de genre vise à argumenter au niveau individuel comme au niveau social le débat autour de la construction du genre et des inégalités qui s'ensuivent. La compétence de genre est un élément fondamental de l'égalité entre femmes et hommes. " (Liebig et al., 2009) Une telle formation pourrait comprendre deux volets: I'un théorique, l'autre pratique. Le volet théorique permettrait d'acquérir un savoir sur la différence entre sexe et genre, et surtout sur le genre comme catégorie sociale, comme ordre symbolique et comme système de hiérarchisation. Ce volet devrait également donner aux enseignants quelques notions de l'histoire de l'éducation des filles, une éducation à la fois limitée, cachée, morale, tardive et empêchée. La partie pratique, quant à elle, permettrait de prendre conscience de ses propres stéréotypes et comportements avec les élèves, par exemple grâce à des enregistrements vidéo. Un programme de formation dans ce sens nous semble être la seule solution pour éviter que les enseignants continuent de perpétuer et de renforcer les stéréotypes de sexe dans une totale innocence, dans une totale inconscience et en toute impunité malgré les déclarations fracassantes que l'on peut entendre au sujet de la politique de l'égalité.

Mais un tel programme bute sur des obstacles institutionnels (manque de volonté politique, manque de moyens), culturels (absence de culture de l'égalité à l'école) et surtout humains car s'il est difficile de sensibiliser les enseignants à cette question, il apparaît plus difficile encore de sensibiliser les jeunes enseignantes en formation qui considèrent la question, d'une part comme dépassée (le « féminisme », c'est I'histoire de leurs mères, voire de leurs grands-mères) et d'autre part comme inutile (tout est déjà acquis). C'est en tout cas ce que montre l'analyse d'un module de sensibilisation à une pédagogie de l'égalité des filles et des garçons à l'école, destiné aux futures enseignantes du préscolaire en Belgique. Questionnées sur leur vécu des discriminations à l'école ou dans la famille, les étudiantes restaient majoritairement silencieuses. Tout au plus étaient-elles capables d'évoquer des différences entre les sexes, sans pouvoir les mettre en rapport avec des inégalités: «Questionnées sur les inégalités dans leur vie quotidienne, les étudiantes n'étaient pas plus prolixes. Ici aussi les traitements discriminatoires sont peu perçus et quand ils le sont, ils sont minimisés (être insultée dans la rue, ce n'est pas vraiment grave) ou, comme dans le cas des sorties le soir ou de la pratique du sport (les deux exemples les plus fréquemment cités), les étudiantes ont tendance à les trouver légitimes pour des raisons qui se réduisent en gros à la faiblesse physique des femmes. " (Plateau, 2007) Les formatrices ont encore constaté chez les étudiantes un mauvais rapport à la théorie. Par exemple, en lisant des extraits de La domination masculine de Bourdieu, elles disaient n'avoir rien appris.

Faut-il tirer de ce sombre constat la conclusion que ces formations ne servent à rien tant la problématique de l'égalité a disparu de l'horizon des jeunes d'aujourd'hui ? Pas tout à fait. L'évaluation du module a montré que les étudiantes avaient appris à mesurer l'ampleur des inégalités de sexe, même si la prise de conscience de leurs propres stéréotypes restait encore très aléatoire. C'est donc sur quatre terrains qu'il faut travailler : prise de conscience à la fois des stéréotypes sexuels véhiculés par l'école et la société, des inégalités, de ses propres stéréotypes et des inégalités dont on est soi-même l'objet.

\section{En conclusion, pensez-vous qu'il faudrait revenir à une école non mixte?}

Comme cela commence à se faire dans certains pays anglo-saxons, vous voulez dire? Cela semble peu probable. Sans doute n'est-ce pas non plus souhaitable, surtout dans un contexte sociopolitique où des demandes de non-mixité surgissent ça et là dans une perspective de ségrégation des sexes et non d'égalité des sexes (concernant l'éducation sportive, par exemple). Ce type de demande ne doit pas nous inciter à proclamer la mixité comme un principe intouchable, mais au contraire nous stimuler pour réfléchir à ses objectifs et à ses effets. II n'est bien évidemment pas question de satisfaire à des exigences de non-mixité dans un but de ségrégation des sexes. II serait en revanche bienvenu de réfléchir, au vu des résultats des recherches scientifiques menées ces trente dernières années, à des expériences pilotes de non-mixité qui ont pour objectif de mieux asseoir l'égalité entre filles et garçons, entre hommes et femmes. Finalement, la situation a peu changé depuis 1996, époque où Claude Zaidman a publié son ouvrage pionnier sur la mixité à l'école primaire. Au lieu de constituer un thème de réflexion, la mixité est un état qui va de soi, ne méritant pas qu'on réfléchisse ni à sa gestion, ni à son bien-fondé. Or tout idéal mérite d'être pensé.

Martine Chaponnière martine.chaponniere@unige.ch Université de Genève 
BAUR E. \& MARTI M. (2000). Kurs auf Gender Kompetenz. Leitfaden für eine geschlechtergerechte Didkatik in der Erwachsenenbildung [Enseigner le genre. Guide pour une didactique respectueuse du genre dans l'éducation des adultes]. Bâle : Gleichstellungsbüro Basel-Stadt.

BUREAU DE L'ÉGALITÉ ENTRE LES FEMMES ET LES HOMMES DU CANTON DE VAUD (2006). L'école de l'égalité. Lausanne: Bureau de l'égalité entre les femmes et les hommes du canton de Vaud. Disponible sur Internet à l'adresse : <http://www.vd.ch/fr/autorites/ departements/dse/bureau-de-legalite/projets/lecolede-legalite/> (consulté le 2 juin 2010).

CHAPONNIÈRE C. \& CHAPONNIÈRE M. (2006). La mixité. Des hommes et des femmes. Gollion [Suisse] : Infolio.

CLIO (2003). "Coéducation et mixité ». Clio, n 18.

DURU-BELLAT M. (1995a). "Filles et garçons à l'école, approches sociologiques et psycho-sociales. $2^{\mathrm{e}}$ partie : La construction scolaire des différences de sexe ". Revue française de pédagogie, n 110, p. 75-109.

DURU-BELLAT M. (1995b). «Garçons et filles à l'école de la différence ». In M. Duru-Bellat, La place des femmes. Paris : La Découverte, p. 600-606.

GROUPE DE RÉFLEXION ET D'ACTION AUTOUR DE LA VIOLENCE (2008). Rapport «Ni Roméos, ni Juliettes?». Une quinzaine sur les violences sexuelles et les relations de genre. Genève: Groupe de réflexion et d'action autour de la violence.

GRÜNEWALD-HUBER E. (1997). Koedukation und Gleichstellung. Eine Untersuchung zum Verhältnis der Geschlechter in der Schule [Égalité et mixité. Enquête sur les comportements des filles et des garçons à l'école]. Coire : Verlag Rüegger.

GUGGENBÜHL A. (2006). Kleine Machos in der Krise [Petits machos en crise]. Fribourg : Herder.

HÉRITIER F. (1996). Masculin/féminin. La pensée de la différence. Paris : Odile Jacob.

HERZOG W., LABUDDE P. \& NEUENSCHWANDER M. (1998). Physik geht uns alle an. Ergebnisse aus der Nationalfondsstudie Koedukation im Physikunterricht [La physique, c'est pour tout le monde. Résultats de l'enquête "Coéducation dans l'enseignement de la physique »]. Berne : Abteilung Pädagogische Psychologie.

LEHMANN H. (2003). Geschlechtergerechter Unterricht. Praxisreflexion von Sprachlehrpersonen [Un enseignement respectueux du genre. Réflexion sur leur pratique par des professeurs de langue]. Bern : Haupt.

LEMMERMÖHLE D. (2001) « Gender und Genderforschung als Herausforderung für die Professionalisierung von Lehrerinnen und Lehrern [Genre et recherche sur le genre: un défi pour la professionnalisation du corps enseignant] ». Beiträge zur Lehrerbildung, $\mathrm{n}^{\circ} 3$, p. 324-334.

LIEBIG B., ROSENKRANZ-FALLEGGER E. \& MEYERHOFER U. (2009). Handbuch Gender-Kompetenz. Ein Praxisleitfaden für (Fach) Hochschulen [Manuel de compétences dans le domaine du genre. Guide pratique pour les universités et les hautes écoles spécialisées]. Zurich: VDF.
MARRO C. \& VOUILLOT F. (2004). "Quelques concepts clefs pour penser et former à la mixité ". Carrefours de l'éducation, $\mathrm{n}^{\circ} 17$, p. 3-21.

MINISTÈRE DE L'EMPLOI, DE LA COHÉSION SOCIALE ET DU LOGEMENT et al. (2006). Convention pour l'égalité entre les filles et les garçons, entre les femmes et les hommes, dans le système éducatif. Disponible sur Internet à l'adresse : <http://media.education.gouv.fr/ file/88/9/3889.pdf> (consulté le $1^{\mathrm{er}}$ mai 2010).

MOSCONI N. (1999). "Les recherches sur la socialisation différentielle des sexes à l'école ». In Y. Lemel \& B. Roudet, Filles et garçons jusqu'à l'adolescence. Paris: L'Harmattan.

MOSCONI N. (2003). " Rapport au savoir et division sociosexuée des savoirs à l'école ". La lettre de l'enfance et de l'adolescence, $\mathrm{n}^{\circ}$ 51, p. 31-38.

PLATEAU N. (2007). " "Donner une voiture aux garçons et une poupée aux filles, on le fait sans s'en rendre compte et je trouve bien qu'on en ait pris conscience". Module de sensibilisation à une pédagogie de l'égalité des filles et garçons à l'école ». In N. Coulon \& G. Cresson, La petite enfance. Entre familles et crèches, entre sexe et genre. Paris : L'Harmattan.

SIBP SCHRIFTENREIHE (2003). " Gendergerecht unterrichten an Berufsschulen [Un enseignement respectueux du genre dans les écoles professionnelles]". SIBP Schriftenreihe, $\mathrm{n}^{\circ} 20$.

SPENCER S., STEELE C. \& QUINN D. (1999). «Stereotype threat and women's math performance ». Journal of Experimental Social Psychology, vol. 35, $\mathrm{n}^{\circ} 1$, p. 4-28.

SPIESS G. \& RENTMEISTER C. (2003). Gender in Lehre und Didaktik. Eine europäische Konferenz in Erfurt [Le genre dans l'enseignement et la didactique. Une conférence européenne à Erfurt]. Berne : Peter Lang.

TRENDBERICHT SKBF (2003). « Keine Lust auf Mathe, Physik, Technik? Zugang zu Mathematik, Naturwissenschaften und Technik attraktiver und geschlechtergerecht gestalten [Pas envie de faire des maths, de la physique et de la technique ? Organiser un accès respectueux du genre aux mathématiques, aux sciences naturelles et à la technique] ». Trendbericht SKBF, $\mathrm{n}^{\circ} 6$.

TRIBUNAL FÉDÉRAL (1982). Arrêt du tribunal fédéral du 12 février 1982, ATF 108, IA 22. Disponible sur Internet à l'adresse : <http://www.bger.ch/fr/index/juridiction/ jurisdiction-inherit-template/jurisdiction-recht/jurisdictionrecht-leitentscheide1954.htm> (consulté le 2 juin 2010).

US DEPARTMENT OF EDUCATION (2005). Single-sex versus coeducational schooling: A systematic review. Washington: US Department of education. Disponible sur Internet à l'adresse: <http://www.ed.gov/rschstat/eval/other/ single-sex/single-sex.pdf $>$ (consulté le $1^{\mathrm{er}}$ mai 2010).

WEST C. \& ZIMMERMAN D. (2009). «Faire le genre ». Nouvelles questions féministes, vol. 28, n० 3 , p. 34-61. [Trad. de F. Malbois de "Doing Gender ». Gender and Society, 1987, n० 1, p. 125-151].

ZAIDMAN C. (1996). La mixité à l'école primaire. Paris: L'Harmattan. 